\title{
Discovering decision algorithm from a distance relay event report
}

\begin{abstract}
In this study rough-set-based data mining strategy was formulated to discover distance relay decision algorithm from its resident event report. This derived algorithm, aptly known as relay CD-prediction rules, can later be used as a knowledge base in support of a protection system analysis expert system to predict, validate or even diagnose future unknown relay events. Nowadays protection engineers are suffering from very complex implementations of protection system analysis due to massive quantities of data coming from diverse points of intelligent electronic devices. In helping the protection engineers deal with this overwhelming data, this study relied merely on digital protective relayôs recorded event report because, among other intelligent electronic devices, digital protective relay sufficiently provided virtually most attributes needed for data mining process in knowledge discovery in database. The method of discovering the distance relay decision algorithm essentially involved formulating rough set discernibility matrix and function from relay event report, finding reducts of pertinent attributes using genetic algorithm and finally generating relay prediction rules. The classification accuracy and the area under the ROC curve measurements provided an acceptable evaluation of the fact that the discovered relay decision algorithm.
\end{abstract}

Keyword: Data mining; Decision table; Digital protective relay; Distance protection; Knowledge discovery in database; Rough set theory 